\title{
Pineal Region Glioblastoma, a Case Report and Literature Review
}

\author{
Hayley Beacher Stowe ${ }^{1}$, C. Ryan Miller ${ }^{2}$, Jing Wu ${ }^{3}$, Dina M. Randazzo ${ }^{4}$ \\ and Andrew Wenhua Ju ${ }^{1 *}$
}

\begin{abstract}
${ }^{1}$ Department of Radiation Oncology, Brody School of Medicine, Greenville, NC, United States, ${ }^{2}$ Department of Pathology, University of North Carolina School of Medicine, Chapel Hill, NC, United States, ${ }^{3}$ Center for Cancer Research National Cancer Institute, Bethesda, MD, United States, ${ }^{4}$ Department of Neuro-Oncology, Duke Health, Durham, NC, United States
\end{abstract}

OPEN ACCESS

Edited by:

Minesh P. Mehta,

Baptist Health South Florida,

United States

Reviewed by:

Michelle Kim,

University of Michigan Health

System, United States

Ari E. Marciscano,

Johns Hopkins University,

United States

*Correspondence:

Andrew Wenhua Ju jua@ecu.edu

Specialty section:

This article was submitted

to Radiation Oncology,

a section of the journal

Frontiers in Oncology

Received: 27 March 2017

Accepted: 26 May 2017

Published: 12 June 2017

Citation:

Stowe HB, Miller CR, WU J,

Randazzo DM and Ju AW (2017)

Pineal Region Glioblastoma, a Case

Report and Literature Review.

Front. Oncol. 7:123.

doi: 10.3389/fonc.2017.00123
Introduction: Pineal region glioblastoma multiforme (GBM) is a rare disease entity with a generally poor prognosis. We present a case of a patient with an unresectable pineal region GBM treated with chemoradiation with favorable outcome.

Case background: A 65-year-old patient who was presented with visual symptoms was found to have a pineal region tumor on imaging. A stereotactic biopsy showed a World Health Organization Grade IV GBM, O-6-methylguanine-DNA methyltransferase (MGMT) promoter methylated, isocitrate dehydrogenase 1 and 2 wild type. The patient was treated with radiotherapy with concurrent temozolomide, followed by adjuvant temozolomide. Disease progression occurred at 58 weeks post-biopsy, which prompted the initiation of bevacizumab. The patient was alive and functioning well as of his last follow up, 166 weeks from the initial biopsy.

Discussion: On our review of the literature, 24 cases of pineal region GBM have been reported. The median reported survival for these previously reported cases was 6 months (range, 2-24 months). This patient has the longest overall survival reported to date for a patient with this diagnosis. This is the first patient in the literature with pineal region GBM who has been reported to have MGMT promoter methylation.

Concluding remarks: Although pineal region GBM is a rare disease entity with a generally poor prognosis, long-term survival is achievable for select patients. MGMT promoter methylation may potentially have prognostic value. Favorable control of recurrent disease with the use of bevacizumab is possible.

Keywords: pineal, glioblastoma, radiotherapy, temozolomide, 0-6-methylguanine-DNA methyltransferase, bevacizumab

\section{INTRODUCTION}

Various tumor histologies can arise in the pineal region, including parenchymal tumors, neuroectodermal tumors, germ cell tumors, and meningeal tumors (1). Gliomas in the pineal region include fibrillary astrocytoma, pilocytic astrocytoma, anaplastic astrocytoma, glioblastoma, oligodendroglioma, ependymoma, and choroid plexus papilloma (2). Glioblastoma multiforme (GBM) is rarely found in the pineal region. This paper presents a case of primary GBM in the pineal region and discusses the clinical course, radiological findings, and treatment approaches with a review

Abbreviations: GBM, glioblastoma multiforme; IMRT, intensity-modulated radiation therapy; MGMT, O-6-methylguanineDNA methyltransferase; IDH, isocitrate dehydrogenase, WHO, World Health Organization. 
of the relevant literature. The patient provided written informed consent for his personal information to be used for research and publication.

\section{CASE BACKGROUND}

A 64-year-old male with no significant past medical history presented with vertical diplopia, headaches for 3 weeks, and 6 months of insomnia. His neurological examination revealed a right cranial nerve IV palsy and gait difficulties. Subsequent CT imaging, 1 month after initial presentation, revealed a poorly marginated, hyperdense mass located in the pineal region. A $\mathrm{CT}$ of the chest, abdomen, and pelvis, and an MRI of the total spine showed no evidence of metastatic disease. MRI of the brain revealed a heterogeneously enhancing pineal mass measuring $2.3 \mathrm{~cm} \times 2.5 \mathrm{~cm} \times 2.3 \mathrm{~cm}$ (Figure 1). The lesion was deemed unresectable due to its location and an image-guided stereotactic needle biopsy was performed. The pathology revealed a highgrade glioma composed of markedly atypical cells, many with giant nuclei and containing abundant mitotic activity (seven mitoses in three high-powered fields), multiple foci of microvascular proliferation, and areas of pseudopalisading necrosis, consistent with GBM, World Health Organization Grade IV (Figure 2). The tumor was positive for O-6-methylguanineDNA methyltransferase (MGMT) methylation and negative for isocitrate dehydrogenase $1 / 2$ mutations. The patient's GBM was treated with concurrent chemoradiation followed by adjuvant chemotherapy $(3,4)$. The patient received intensity-modulated radiation therapy consisting of $60 \mathrm{~Gy}$ in 2 Gy daily fractions with concurrent temozolomide at $75 \mathrm{mg} / \mathrm{m}^{2}$. The initial fields were treated to a dose of $50 \mathrm{~Gy}$ and encompassed a $2 \mathrm{~cm}$ margin along white matter tracts from the enhancing tumor and the surrounding edema, and also covered the third and fourth ventricles, lateral ventricles, cerebral aqueduct, tectum, partial thalamus, and partial brainstem in the clinical target volume. The majority of the lateral ventricles were covered but the extreme ends of the horns were excluded due to concerns of additional toxicity, given the volume of brain treated, the prepontine cistern was not specifically targeted. An effort was made to cover the majority of the ventricles based on prior clinical reports describing a propensity for leptomeningeal spread of disease for pineal region GBMs. This initial radiotherapy plan was followed by a boost of $10 \mathrm{~Gy}$ to the enhancing tumor with a $2 \mathrm{~cm}$ margin along white matter tracts. The combined radiotherapy plan is displayed in Figure 3. The patient was placed on dexamethasone during the course of radiotherapy due to concerns that radiotherapy may potentially worsen local edema which could then result in obstruction of the cerebral aqueduct.

The patient developed alopecia and Common Terminology Criteria for Adverse Events version 4 Grade 1 dermatitis during the course of chemoradiation, but otherwise tolerated his concurrent chemoradiation well. Daily CT scans were used for radiotherapy image guidance and showed no signs of hydrocephalus developing during treatment. An MRI 4 weeks after completion of radiotherapy showed a slight decrease in the size of the pineal GBM to $2.3 \mathrm{~cm} \times 2.0 \mathrm{~cm} \times 1.9 \mathrm{~cm}$. He continued on maintenance
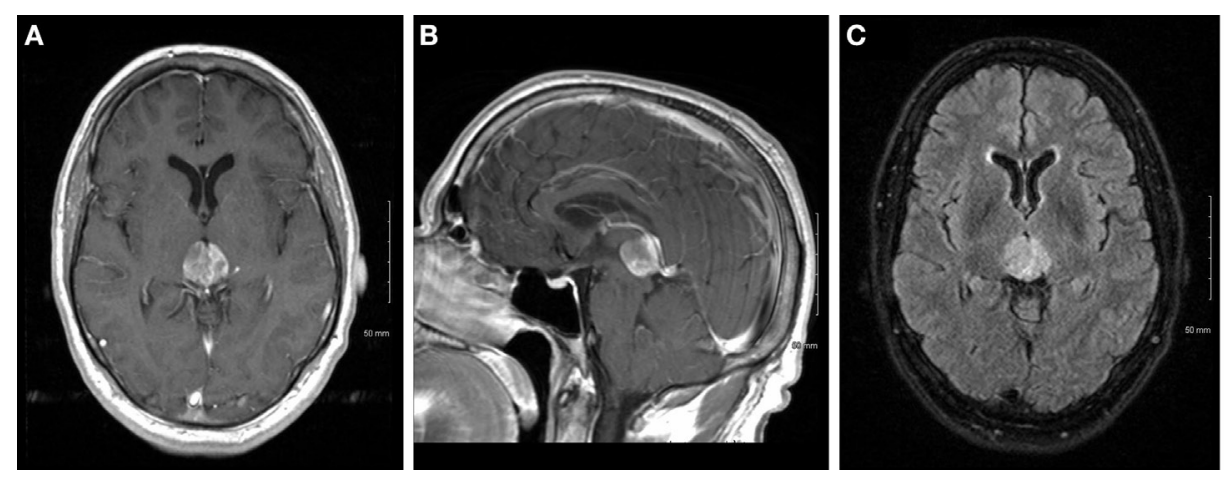

FIGURE 1 | Axial (A) and sagittal (B) T1 post-contrast and T2 fluid attenuated inversion recovery (C) images of the tumor at initial presentation.

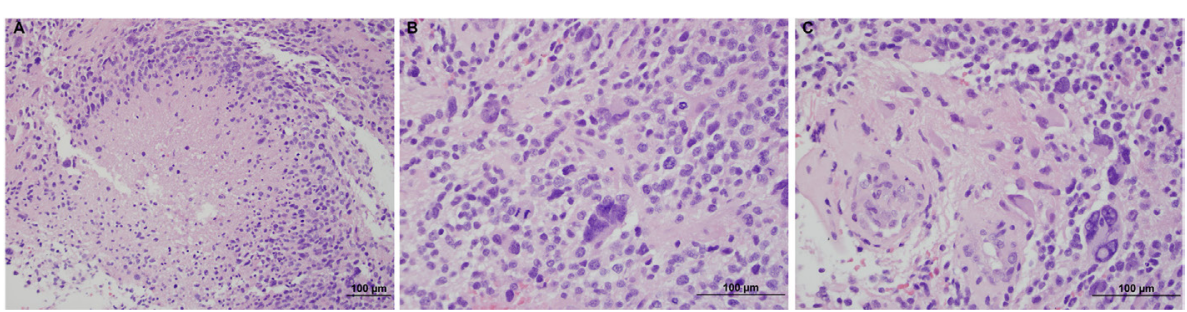

FIGURE 2 | Hematoxylin and eosin stains of the tumor, showing necrosis at $20 \times(\mathbf{A})$, mitotic figures at $40 \times(\mathbf{B})$, and microvascular proliferation at 40× (C) 

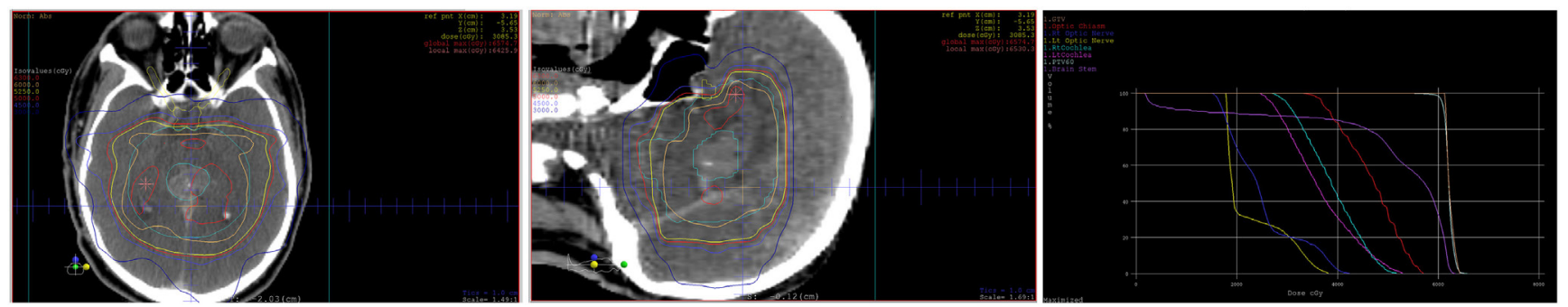

FIGURE 3 | Axial and sagittal views of the dose distribution of the radiotherapy plan with the dose-volume histogram.
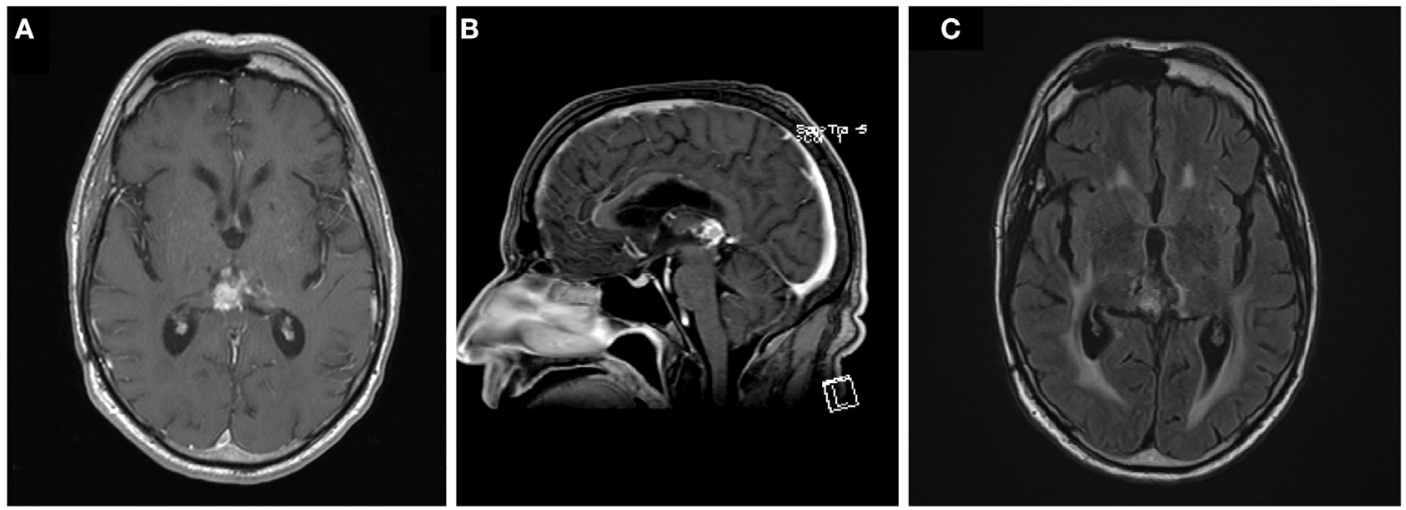

FIGURE 4 | Axial (A) and sagittal (B) T1 post-contrast and T2 fluid attenuated inversion recovery (C) images of the tumor at last follow up, 166 weeks following the initiation of chemoradiation.

therapy with temozolomide, 5 days out of every 28 days per cycle, at $150 \mathrm{mg} / \mathrm{m}^{2}$ for the first cycle and increased to $200 \mathrm{mg} / \mathrm{m}^{2}$ for subsequent cycles.

An MRI was performed at 58 weeks post-biopsy that showed two new lesions in the brainstem and parietal lobe, both within the prior radiotherapy fields. A total spine MRI did not reveal any drop metastases, nor was there any indication of CSF dissemination per lumbar puncture. Bevacizumab $7.5 \mathrm{mg} / \mathrm{kg}$ every 3 weeks was added to the temozolomide therapy (5). Temozolomide was discontinued after 17 cycles, a total of 88 weeks after the biopsy. Due to clinical symptoms of worsening gait disturbance, which corresponded to MRI findings suspicious for infarct within the radiotherapy fields, at 129 weeks post-biopsy, bevacizumab was discontinued and serial MRIs were subsequently performed every 2 months to closely monitor his disease. The MRI performed 146 weeks after the initial biopsy, when compared over multiple prior studies dating back to 111 weeks post-biopsy, showed subtle, progressive increase in size of the pineal mass from $11 \mathrm{~mm} \times 11 \mathrm{~mm}$ to $14 \mathrm{~mm} \times 11 \mathrm{~mm}$ and a confluent periventricular white matter T2/fluid attenuated inversion recovery hyperintensity that is non-specific and stable, this was redemonstrated in his MRI at 166 weeks after biopsy (Figure 4).

Clinically, the patient continues to have double vision, which is corrected by prism glasses. The diplopia continues to cause gait disturbance, which has improved with physical therapy and the patient no longer requires the use of a cane. He was last seen in clinic 166 weeks following his biopsy and 156 weeks following the initiation of chemoradiation for a follow up. He was continuously monitored closely off systemic therapy.

\section{DISCUSSION}

Tumors of the pineal region are uncommon intracranial neoplasms. The most common glioma in the pineal region is a welldifferentiated astrocytoma (1). GBM, though common in the brain as a whole, is rarely seen in the pineal region. Since the first report on primary pineal GBM by Bradfield and Perez in 1972, 24 case reports have been described (6-23) (Table 1).

The clinical symptoms associated with the presentation of a pineal GBM are associated with compression of adjacent structures. Of the 24 patients described in literature, $66.7 \%$ presented with signs or symptoms of increased intracranial pressure, and $41.7 \%$ also presented with visual disturbances including diplopia, nystagmus, and blurry vision, which may be associated with Parinaud's syndrome. CT scans of pineal GBM typically show heterogeneously contrast-enhancing masses with zones of low density. Pineal region GBM is shown to infiltrate the leptomeninges with $33.3 \%$ of patients in the literature.

Of the 24 cases in the literature, $54.1 \%$ have a survival duration of less than 11 months, and $70.8 \%$ having a survival duration of less than 24 months. Of the 18 patients where survival was reported (excluding our current patient), the median 
TABLE 1 | Summary of published cases of pineal region glioblastoma

\begin{tabular}{|c|c|c|c|c|c|c|c|}
\hline Reference & Age & Sex & Symptoms & Radiographic findings & $\begin{array}{l}\text { Leptomeningeal } \\
\text { dissemination }\end{array}$ & Treatment & Survival \\
\hline Bradfield et al. (6) & 53 & $\mathrm{~F}$ & N/A & $\mathrm{HCP}$, mass in posterior third ventricle & No (autopsy) & Resection & $\begin{array}{l}\text { Post-operative } \\
\text { death }\end{array}$ \\
\hline Bradfield et al. (6) & 5 & $\mathrm{~F}$ & N/A & $\mathrm{HCP}$, mass in posterior third ventricle & No (autopsy) & Shunt & 27 months \\
\hline DeGirolami et al. (8) & 3 cases & & Increased ICP, vertical gaze palsy in one & $N / A$ & N/A & $\begin{array}{l}\text { RT for all three cases, } \\
\text { resection for one }\end{array}$ & N/A \\
\hline Kalyanaraman (12) & 68 & $\mathrm{~F}$ & $\begin{array}{l}\text { Ataxia, confusion, urinary incontinence, } \\
\text { upgaze limitation }\end{array}$ & CT: HCP, calcified midline mass & N/A & Resection, RT & 4 months \\
\hline Norbut et al. (13) & 36 & $\mathrm{~F}$ & HA, blurry vision, Parinaud's Syndrome & CT: HCP, mass in posterior third ventricle & Yes & Shunt, RT & 4 months \\
\hline Frank et al. (10) & 52 & $\mathrm{~F}$ & Intracranial ICP, oculomotor disturbances & $\mathrm{HCP}$, mass in third ventricle & N/A & Stereotactic biopsy, RT & 4 months \\
\hline Edwards et al. (9) & 12 & $\mathrm{~F}$ & N/A & $N / A$ & N/A & Resection, RT, chemo & 18 months \\
\hline Vaquero et al. (17) & 63 & $\mathrm{~F}$ & HA, behavioral changes & $\begin{array}{l}\text { CT: rounded hyperdense, mass with ring } \\
\text { enhancement }\end{array}$ & N/A & $\begin{array}{l}\text { Shunt, resection, whole } \\
\text { brain RT }\end{array}$ & 6 months \\
\hline Pople et al. (14) & 6 & $\mathrm{~F}$ & $\begin{array}{l}\text { HA, N/N, diplopia, decreased visual acuity, } \\
\text { CN VI palsy, upgaze limited }\end{array}$ & $\mathrm{CT}$ and MRI: $\mathrm{HCP}$, enhancing mass & Yes & $\begin{array}{l}\text { Shunt, resection, local RT, } \\
\text { chemo }\end{array}$ & 4 months \\
\hline Cho et al. (7) & 63 & M & Increased ICP, changing behavior & $\mathrm{HCP}$, hyperdense pineal mass with enhancement & N/A & Resection, RT & 6 months \\
\hline Gasparetto et al. (11) & 29 & $\mathrm{~F}$ & HA, drowsiness, fever, dizziness, seizure & $\begin{array}{l}\mathrm{CT} \text { and MRI: ill-defined heterogeneously enhanced mass } \\
\text { with extension to thalamus }\end{array}$ & No & Shunt, resection & 2 months \\
\hline Toyooka et al. (15) & 49 & M & HA, diplopia, memory disturbance & MRI: HCP, irregular heterogeneously enhanced mass & Yes & $\begin{array}{l}\text { Shunt, resection, chemo } \\
\text { (ACNU), local RT }\end{array}$ & 11 months \\
\hline Amini et al. (16) & 40 & M & HA, N/N, diplopia, blurry vision & $\begin{array}{l}\text { CT: obstructive HCP, strong enhancement, punctuate } \\
\text { calcification MRI: heterogeneously enhancing with } \\
\text { central necrosis, extension into midbrain }\end{array}$ & Yes & $\begin{array}{l}\text { Endoscopic TVB, resection, } \\
\text { shunt, whole brain RT, chemo } \\
\text { (temozolomide) }\end{array}$ & 5 months \\
\hline Amini et al. (16) & 43 & M & $\begin{array}{l}\text { Ha, disequilibrium, decreased level of } \\
\text { mental status }\end{array}$ & MRI: heterogeneously enhancing, $\mathrm{HCP}$ & Yes & $\begin{array}{l}\text { TVB, resection, whole } \\
\text { brain } \mathrm{RT} \text {, chemo }\end{array}$ & 7 months \\
\hline Amini et al. (16) & 52 & $\mathrm{~F}$ & HA, N/N, diplopia, blurry gaze palsy & $\begin{array}{l}\text { MRI: heterogeneously enhancing with central } \\
\text { necrosis, obstructive HDC }\end{array}$ & Yes & Endoscopic TVB, RT & 2 months \\
\hline Moon et al. (20) & 68 & M & $\mathrm{HA}, \mathrm{N} / \mathrm{N}$, ataxia & $\begin{array}{l}\mathrm{CT} \text { : } \mathrm{HCP} \text {, hypodense mass MRI: irregular heterogeneously } \\
\text { ring-enhanced mass with central necrosis }\end{array}$ & Yes & Resection, shunt & 2 months \\
\hline Ozgural et al. (18) & 60 & M & $\mathrm{HA}$, ataxia & $\begin{array}{l}\text { CT: triventricular HCP, isodense rounded mass MRI: } \\
\text { heterogeneous, contrast enhanced mass }\end{array}$ & N/A & $\mathrm{RT}$, chemo & 24 months \\
\hline Mansour et al. (21) & 69 & M & Altered mental status, vertigo & $\mathrm{CT}$ and MRI: heterogeneous mass, $\mathrm{HCP}$ & N/A & Biopsy, chemo, RT & 16 months \\
\hline Suzuki et al. (22) & 65 & M & Disturbed consciousness & $\mathrm{CT}$ : intraventricular hemorrhage, $\mathrm{HCP}$ & N/A & Resection, RT, chemo & N/A \\
\hline Sugita et al. (23) & 52 & $\mathrm{~F}$ & HA, memory disturbance & MRI: mass in pineal, $\mathrm{HCP}$ & N/A & Resection, RT, chemo & 24 months \\
\hline Sugita et al. (23) & 18 & M & HA, CN VI palsy & MRI: mass in the pineal, $\mathrm{HCP}$ & $\mathrm{N} / \mathrm{A}$ & Resection, RT, chemo & 13 months \\
\hline Liu et al. (19) & 30 & M & HA, vomiting, LE numbness & $\begin{array}{l}\text { MRI: multifocal lesion largest at the right } \\
\text { thalamus }\end{array}$ & N/A & Resection, RT, chemo & N/A \\
\hline Present case & 65 & M & Right CN IV palsy with gait difficulties & СТ: heterogeneous mass & N/A & Biopsy, chemo, RT & $>38$ months \\
\hline
\end{tabular}


survival was 6 months (range, 2-24 months). Only one other patient has survived longer than 2 years. The maximum survival duration documented is the case presented in this report, with a survival time of 38 months at the time of manuscript preparation.

Pineal GBM is generally associated with a poor prognosis. Of the three cases that were treated with radiotherapy alone, the median survival was 3.5 months (range, $0-4$ months) $(10,13,16)$. The three patients in the literature treated with surgery alone had a median survival of 1.5 months, with a maximum survival of 2 months $(6,11,20)$. Patients treated with resection followed by radiotherapy had a slightly higher median survival of 5.5 months (range, 4-6 months) $(7,12,17)$. A tri-modal treatment approach of surgical resection followed by chemotherapy and radiotherapy yielded a median survival time of 12 months (range, 4-24 months) among the cases reported thus far in the literature $(9,14-16$, $21,23)$. However, those patients who received radiotherapy and chemotherapy without resection, including the present case, had the highest median survival duration of 20 months (range,

7-36 months) $(16,18,21)$.

It is unknown why patients who did not undergo resection appear to have had a more favorable outcome in this review, indeed the difficulty of achieving a gross total resection in tumors located in this region is thought to be one of the factors that contribute to the poor prognosis of tumors in this region $(7,18$, 20,21 ), in addition to the propensity for pineal region GBMs to exhibit leptomeningeal spread of disease $(13,16,21,24)$, and the fact that progressive disease in this region could quickly affect critical structures in proximity to the pineal gland $(16,24)$. It may be possible that any attempt at resection could increase the risk of leptomeningeal seeding, and perhaps coverage of a significant volume of the ventricles in the radiotherapy plan as was done in this patient could aid in sterilizing leptomeningeal disease, but these hypotheses need to be studied in more detail.

O-6-methylguanine-DNA methyltransferase promoter methylation predicts for better outcomes for patients with GBM in other locations in the brain, including patients with an unresectable GBM (24). Our patient's favorable outcome is likely associated with his MGMT promoter methylation status. To our knowledge, this is the first reported pineal GBM with MGMT promoter methylation. In prior literature, the methylation status was either not reported or was negative. A comparison of survival times of patients treated with chemoradiation based on MGMT promotor status would be valuable once more cases are reported, the results may lend more support to the premise that reasonable survival outcomes can be achieved with chemoradiation in

\section{REFERENCES}

1. Hirato J, Nakazato Y. Pathology of pineal region tumors. J Neurooncol (2001) 54:239-49. doi:10.1023/A:1012721723387

2. RingertzN, Nordenstam H, Flyger G. Tumors of the pineal region.J Neuropathol Exp Neurol (1954) 13:540-61. doi:10.1097/00005072-195410000-00004

3. Stupp R, Hegi ME, Mason WP, van den Bent MJ, Taphoorn MJ, Janzer RC, et al. Effects of radiotherapy with concomitant and adjuvant temozolomide versus radiotherapy alone on survival in glioblastoma in a randomized phase
MGMT promoter methylated patients in the absence of gross total resection.

Based on the literature review performed for this report, there is no reported utilization of bevacizumab in patients with pineal region GBM. Our patient had an excellent response to bevacizumab with good long-term control of disease after initial progression, which has contributed to his good overall survival. This favorable response reflects data seen in cases of recurrent GBM outside of the pineal region (5).

\section{CONCLUDING REMARKS}

Pineal GBM is a rare disease that is associated with a poor prognosis. The majority of patients present with signs of increased intracranial pressure and visual symptoms. It is difficult to draw conclusions from the small number of total patients reported over a number of decades with differing treatment paradigms. This is the first pineal region GBM that reports positive MGMT promoter methylation and use of bevacizumab upon progression. Our case suggests that durable control of the disease can potentially be achieved in the absence of a gross total surgical resection. Further research in the role of MGMT methylation as a prognostic indicator and of the response of pineal region GBM to bevacizumab is warranted.

\section{ETHICS STATEMENT}

The patient described in this case report consented to have his case described in this manuscript. Written permission was obtained from the patient's treating medical oncologist for publication. The patient's personal identifiers were not included in this manuscript.

\section{AUTHOR CONTRIBUTIONS}

HS: primary author of manuscript. CM: provided comments regarding case pathology with figures. JW: provided comments regarding patient's systemic therapy and associated toxicities. DR: provided comments regarding patient's systemic therapy and associated toxicities, provided radiographs. AJ: assisted primary author in manuscript preparation. Provided information regarding patient's radiotherapy.

\section{FUNDING}

This manuscript did not have any sources of funding.

III study: 5 year analysis of the EORTC/NCIC trial. Lancet Oncol (2009) 10:459-66. doi:10.1016/S1470-2045(09)70025-7

4. Hegi ME, Liu L, Herman JG, Stupp R, Wick W, Weller M, et al. Correlation of O6-methylguanine methyltransferase (MGMT) promoter methylation with clinical outcomes in glioblastoma and clinical strategies to modulate MGMT activity. J Clin Oncol (2008) 26(25):4189-99. doi:10.1200/ JCO.2007.11.5964

5. Brandes AA, Finocchiaro G, Zagonel V, Reni M, Fabi A, Caserta C, et al. Early tumour shrinkage as a survival predictor in patients with recurrent 
glioblastoma treated with bevacizumab in the AVAREG randomized phase II study. Oncotarget (2017). doi:10.18632/oncotarget.15735

6. Bradfield JS, Perez CA. Pineal tumors and ectopic pinealomas. Analysis of treatment and failures. Radiology (1972) 103:399-406. doi:10.1148/103.2.399

7. Cho BK, Wang KC, Nam DH, Kim DG, Jung HW, Kim HJ, et al. Pineal tumors: experience with 48 cases over 10 years. Childs Nerv Syst (1998) 14:53-8. doi:10.1007/s003810050175

8. DeGirolami U, Schmidek H. Clinicopathological study of tumors of the pineal region. J Neurosurg (1973) 39:455-62. doi:10.3171/jns.1973.39.4.0455

9. Edwards MS, Hudgins RJ, Wilson CB, Levin VA, Wara WM. Pineal region tumors in children. JNeurosurg (1988) 68:689-97. doi:10.3171/ jns.1988.68.5.0689

10. Frank F, Gaist G, Piazza G, Ricci RF, Sturiale C, Galassi E. Stereotaxic biopsy and radioactive implantation for interstitial therapy of tumors of the pineal region. Surg Neurol (1985) 23:275-80. doi:10.1016/0090-3019(85)90095-3

11. Gasparetto EL, Warszawiak D, Adam GP, Bleggi-Torres LF, de Carvalho Neto A. Glioblastoma multiforme of the pineal region: case report. Arq Neuropsiquiatr (2003) 61:468-72. doi:10.1590/S0004-282X2003000300027

12. Kalyanaraman UP. Primary glioblastoma of the pineal gland. Arch Neurol (1979) 36:717-8. doi:10.1001/archneur.1979.00500470087020

13. Norbut AM, Mendelow H. Primary glioblastoma multiforme of the pineal region with leptomeningeal metastases: a case report. Cancer (1981) 47:592-6. doi:10.1002/1097-0142(19810201)47:3<592::AID-CNCR2820470328>3.0. CO;2-G

14. Pople IK, Arango JC, Scaravilli F. Intrinsic malignant glioma of the pineal gland. Childs Nerv Syst (1993) 9:422-4. doi:10.1007/BF00306198

15. Toyooka T, Miyazawa T, Fukui S, Otani N, Nawashiro H, Shima K. Central neurogenic hyperventilation in a conscious man with CSF dissemination from a pineal glioblastoma. J Clin Neurosci (2005) 12:834-7. doi:10.1016/j. jocn.2004.09.027

16. Amini A, Schmidit R, Salzman K, Chin S, Couldwell WT. Glioblastoma multiforme of the pineal region. J Neurooncol (2006) 79:307-14. doi:10.1007/ s11060-006-9145-x
17. Vaquero J, Ramiro J, Martinez R. Glioblastoma multiforme of the pineal region. J Neurosurg Sci (1990) 34:149-50.

18. Ozgural O, Kahilogullari G, Bozkurt M, Heper AO, Savas A. Primary pineal glioblastoma: a case report. Turk Neurosurg (2013) 23:572-4. doi:10.5137/10195149.JTN.5994-12.1

19. Liu Y, Hao S, Yu L, Gao Z. Long-term temozolomide might be an optimal choice for patient with multifocal glioblastoma, especially with deep-seated structure involvement: a case report and literature review. World J Surg Oncol (2015) 13:142. doi:10.1186/s12957-015-0558-x

20. Moon KS, Jung S, Jung TY, Kim IY, Lee MC, Lee KH. Primary glioblastoma in the pineal region: a case report and review of the literature. J Med Case Rep (2008) 2:288. doi:10.1186/1752-1947-2-288

21. Mansour J, Fields B, Macomson S, Rixe O. Significant anti-tumor effect of bevacizumab in treatment of pineal gland glioblastoma multiforme. Target Oncol (2014) 9:395-8. doi:10.1007/s11523-014-0327-8

22. Suzuki R, Suzuki K, Sugiura Y, Takano I, Nagaishi M, Shimizu N, et al. A case of glioblastoma multiforme in the pineal region with intraventricular hemorrhage. No Shinkei Geka (2014) 42(5):429-35.

23. Sugita Y, Terasaki M, Tanigawa K, Ohshima K, Morioka M, Higaki K, et al. Gliosarcomas arising from the pineal gland region uncommon localization. Neuropathology (2016) 36(1):56-63. doi:10.1111/neup.12226

24. Thon N, Thorsteinsodttir J, Eigenbrod S, Schuller U, Lutz J, Kreth S, et al Outcome in unresectable glioblastoma: MGMT promoter methylation makes the difference. J Neurol (2017) 264(2):350-8. doi:10.1007/s00415-016-8355-1

Conflict of Interest Statement: The authors declare that the research was conducted in the absence of any commercial or financial relationships that could be construed as a potential conflict of interest.

Copyright $\odot 2017$ Stowe, Miller, Wu, Randazzo and Ju. This is an open-access article distributed under the terms of the Creative Commons Attribution License (CC BY). The use, distribution or reproduction in other forums is permitted, provided the original author(s) or licensor are credited and that the original publication in this journal is cited, in accordance with accepted academic practice. No use, distribution or reproduction is permitted which does not comply with these terms. 